\title{
A COGNITIVE FINGERPRINT IN HUMAN RANDOM NUMBER GENERATION
}

\author{
Marc-André Schulz ${ }^{1, *}$, Sebastian Baier ${ }^{2}$, Benjamin Böhme ${ }^{2}$, Danilo Bzdok ${ }^{3}$, and Karsten Witt ${ }^{4}$ \\ ${ }^{1}$ Department of Psychiatry and Psychotherapy, RWTH Aachen University, Aachen, Germany \\ ${ }^{2}$ Department of Neurology, Christian-Albrecht-University Kiel, Kiel, Germany \\ ${ }^{3}$ Department of Biomedical Engineering, Faculty of Medicine, McGill University, Montreal, Canada \\ ${ }^{4}$ Department of Neurology and Research Center Neurosensory Science, Carl von Ossietzky University, \\ Oldenburg, Germany \\ *Correspondence: marc.schulz@rwth-aachen.de
}

Most work in the neurosciences collapses data from multiple subjects to obtain robust statistical results. This research agenda ignores that even in healthy subjects brain structure and function are known to be highly variable [1]. Recently, Finn and colleagues showed that the brain's functional organisation is unique to each individual and can yield human-specific connectome fingerprints [2]. This raises the question whether unique functional brain architecture may reflect a unique implementation of cognitive processes and problem solving - i.e. "Can we identify single individuals based on how they think?".

The present study addresses the general question of interindividual differences in the specific context of human random number generation. We analyzed the deployment of recurrent patterns in the pseudorandom sequences to develop an identification scheme based on subject-specific volatility patterns. We demonstrate that individuals can be reliably identified based on how they how they generate randomness patterns alone. We moreover show that this phenomenon is driven by individual preference and inhibition of patterns, together forming a cognitive fingerprint.

In the Random Number Generation (RNG) Task, subjects verbally produce a random sequence of digits. Based on the premise that variations in executive functions are reflected in the characteristic ways in which humans deviate from mathematical randomness, random number generation has previously been used to investigate cognitive changes in brain disorders, such as in Parkinson's disease and schizophrenia $[3,4]$. The random generation process can be characterized by certain predominant patterns (n-tuples, e.g. "4-3-2-1" is a length 4 pattern). These occur in different variations ("3-4-2-1" is a variation of the former) repeatedly throughout the sequence [5]. Variation-tolerant search based on the Damerau-Levenshtein distance measures the prevalence of a pattern in a sequence and identifies predominant patterns. Knowledge of the predominant patterns allows predicting individual upcoming digits in the running sequence.

130 healthy adults (53 male, aged 35.3 years $(\mathrm{SD}=15.6)$ generated two individual sessions of random sequences. These were composed of the digits „1“ to „9“, 300 digits long, paced by a $1 \mathrm{~Hz}$ metronome, with 40 minutes pause between the sessions. 20 subjects generated an additional sequence after one week. The concept of randomness was introduced by analogy to a fair die and to drawing numbered balls from a bag with replacement. Subjects were instructed to implement an uniform distribution, independence of choices, and to avoid patterns and algorithms, i.e. to create an unpredictable sequence. 
Our variation-tolerant search computes the prevalence of a pattern in a sequence. Thus we can characterize sequences by their length-n patterns and consider any random sequence as a point in a $9^{n}$ dimensional vector space. The Euclidean distance of these points gives an intuitive measure of their similarity. Using this metric of sequence-sequence similarity, we achieved an identification performance (AUC) of $(96.48 \pm 0.69) \%$. That is, the algorithm correctly ranked a randomly chosen pair of sequences originating from the same subject higher than a randomly chosen pair from different subjects in 96 out of 100 cases) for length- 6 patterns. Identification performance increased with pattern length $(\mathrm{z}=4.67$; $\mathrm{p}<0.001)$ and did not significantly change $(\mathrm{z}=0.87$; $\mathrm{p}=0.62)$ over the course of one week.

These statistical analyses prompt two fundamental conclusions. First, people have unique digit preferences, either as preferences in and of themselves or as a result of unique generative algorithms. Second, higher-order patterns are a unique feature of individuals and contribute to identification success.

To detail this effect, all $9^{3}$ length-3 patterns were analyzed for their prevalence in each of the sequences. For each sequence, patterns were sorted from most common to most rare. Then, the difference in affinity for sequences of the same subject vis-à-vis all other subjects was calculated for each of these patterns. This allowed for comparing the degree of individuality (i.e. the difference between inter- and intra-subject affinity differences) for rare and common patterns (Figure 1). Clearly visible maxima existed for both strongly preferred and strongly inhibited patterns. We identified four areas of interest: a) Universal exceptions - Certain patterns are universally agreed to be subjectively non-random (e.g. triplets of the same digit). b) Rare patterns - Duplets and x-y-x patterns; their degree of individuality is dominated by individual single digit preferences. c) Individual inhibitions - Subjects individually inhibit certain patterns. d) Individual preferences - subjects employ several highly over-represented person-specific "predominant patterns". One might expect to also see universal preferences. These were likely mixed in with the individual preferences and therefore not clearly distinguishable in this figure.

We conclude that the mechanism by which humans generate random sequences is a) highly unique and that $b$ ) this uniqueness is driven by both individual preferences and individual inhibitions. This insight begs further questions: a) Do we really see unique generative algorithms or can our results be explained by individual differences in cognitive capacity? The latter is unlikely, as the few relevant components of cognitive capacity (e.g. working memory) should only affect correspondingly few dimensions of randomness. It cannot explain the entire effect of cognitive individuality. b) Where do the individual preferences and inhibitions originate? They could be familiar patterns from the subjects personal environment: Based on the degree of familiarity, subjects might either perceive these patterns as non-random and suppress them, or habitually produce them preferentially ("environmental cognitive fingerprint"). But tests for the individual telephone numbers, postal codes, and birthdates revealed no significant anomalies, leading us to conclude that individuality in RNG is most likely the direct result of an individual generative algorithm ("procedural cognitive fingerprint").

Our experimental and statistical techniques enable simultaneous tracking of inhibitory (individual pattern inhibitions) and generative (pattern preferences) performance. This may be a useful tool for investigating attention-deficit/hyperactivity disorder, pharmacological interventions, or consequences of sleep deprivation. Moreover, it underlines the notion that human cognition does not only vary cross-culturally [6], but that the cognitive strategies and approaches people take to solve specific problems are fundamentally different from individual to individual. 

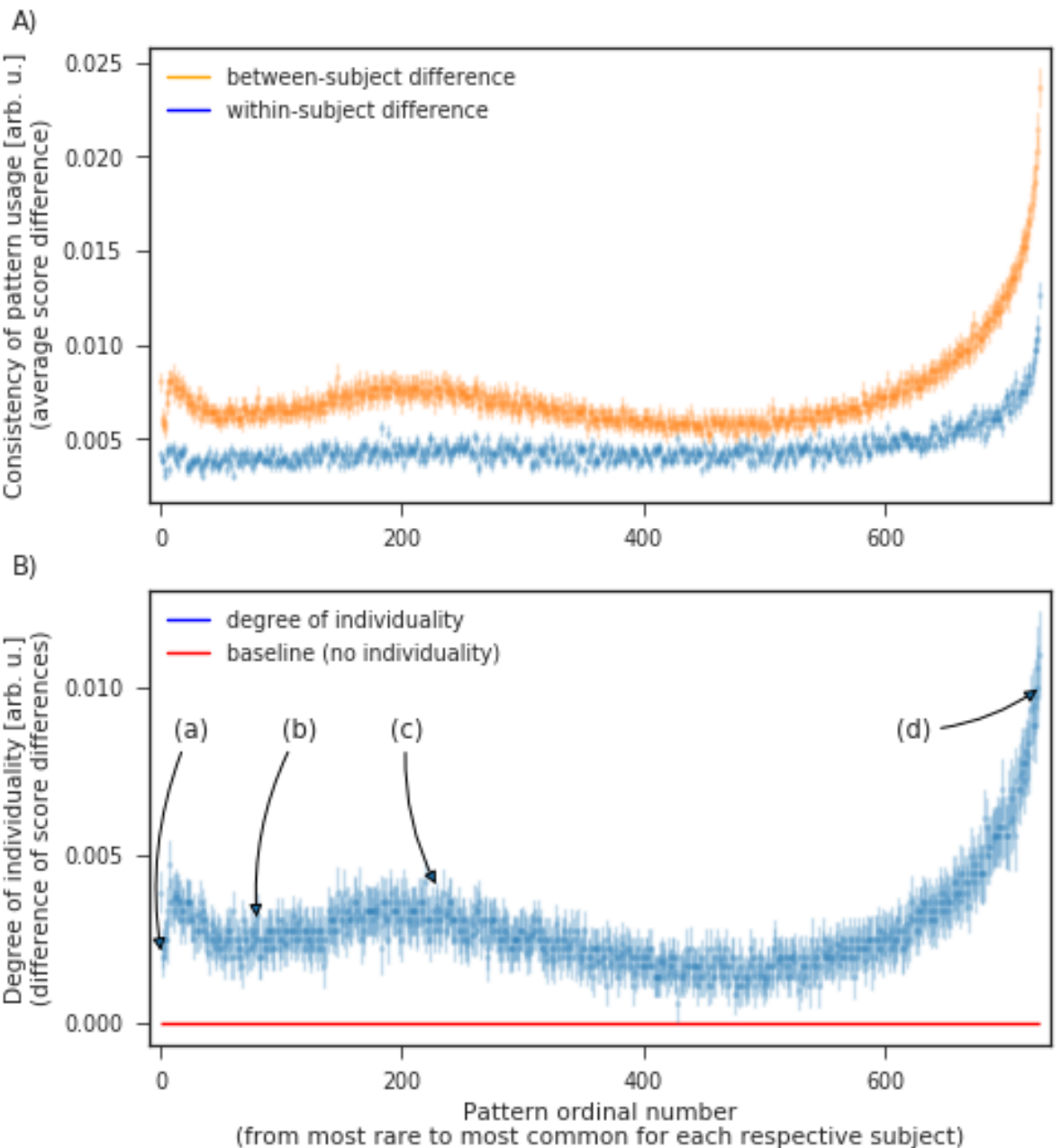

Figure 1: A) Inter- and intra-subject difference in usage of all $9^{3}$ length-3 patterns. The ordinal numbers represent each subject's respective most rare to most common patterns. B) The difference between interand intra-subject differences represents the degree of individuality for the respective rare and common patterns. The marked areas represent a) universal exceptions, b) rare patterns, c) individual inhibitions, d) individual preferences. All error bars represent SEM. 


\section{Author Contributions}

MAS and KW designed the study. MAS, DB and KW analysed the data and wrote the paper. SB and BB performed the experiments.

\section{Declaration of Interests}

The authors declare no competing financial interests

\section{References}

1. Kanai R, Rees G. The structural basis of inter-individual differences in human behaviour and cognition. Nat Rev Neurosci. 2011;12: 231-242.

2. Finn ES, Shen X, Scheinost D, Rosenberg MD, Huang J, Chun MM, et al. Functional connectome fingerprinting: identifying individuals using patterns of brain connectivity. Nat Neurosci. 2015;18: $1664-1671$.

3. Brown RG, Soliveri P, Jahanshahi M. Executive processes in Parkinson's disease--random number generation and response suppression. Neuropsychologia. 1998;36: 1355-1362.

4. Rosenberg S, Weber N, Crocq MA, Duval F, Macher JP. Random number generation by normal, alcoholic and schizophrenic subjects. Psychol Med. Cambridge University Press; 1990;20: 953-960.

5. Schulz M-A, Schmalbach B, Brugger P, Witt K. Analysing humanly generated random number sequences: a pattern-based approach. PLoS One. 2012;7: e41531.

6. Cole M. The Cultural Context of Learning and Thinking: An Exploration in Experimental Anthropology. First Edition. Basic Books; 1971. 